\title{
COVID-19 lock-down significantly reduced number of surgical presentations in an emergency department
}

\author{
René Fahrner (ID · Stefan Bähler · Gregor Lindner
}

Received: 15 September 2020 / Accepted: 8 January 2021 / Published online: 28 January 2021

(c) Springer-Verlag GmbH, AT part of Springer Nature 2021

\begin{abstract}
Summary
Background Coronavirus disease 2019 (COVID-19) is an acute virus infection, which was declared by the World Health Organization (WHO) as a pandemic. As a consequence, the Swiss government decreed a public lock-down to reduce and restrict further infections. The aim of this investigation was to provide the impact of the COVID-19 lock-down on the presentations of patients in an interdisciplinary emergency department (ED).

Patients, material and methods A retrospective study was performed at an interdisciplinary Swiss ED during the lock-down period. All patients who presented to the ED were enrolled in this investigation and compared to a control cohort during the same time period in 2019. Data regarding patients' characteristics, medical specialty, time course of presentations during the observation period, outpatient or hospital admission were analyzed.

Results In total 7072 patients were included in the final analysis. During the lock-down period the number of ED presentations significantly decreased by $29 \%$ in comparison to the control period in the previous year $(p<0.0001)$. The analysis of the time course revealed that in each week the number of patients was lower with $13-43 \%$ reduction. Irrespective of the medical specialty, the number of presentations decreased during the COVID-19 situation, whereas this decrease was
\end{abstract}

\section{R. Fahrner, M.D. $(\bowtie) \cdot$ S. Bähler}

Department of General, Visceral and

Thoracic Surgery, Bürgerspital Solothurn,

Schöngrünstraße 42, 4500 Solothurn, Switzerland

r.fahrner@web.de

G. Lindner, M.D.

Department of General Internal and Emergency Medicine,

Bürgerspital Solothurn, Solothurn, Switzerland higher in surgical diseases $(31 \%, p<0.0001)$ than in non-surgical presentations $(3.6 \%, p=0.4)$.

Conclusion The socioeconomic lock-down was associated with reduced number of presentations in an ED. Mainly, surgical diseases and injuries were affected in terms of a reduction of presentations during the COVID-19 lock-down period.

Keywords COVID-19 • Emergency department . Medicine $\cdot$ Surgery $\cdot$ Pandemic

\section{Introduction}

Coronavirus disease 2019 (COVID-19) is an acute virus infection of the respiratory tract with reported mild to severe courses $[1,2]$. In contrast to severe acute respiratory syndrome (SARS), which was caused by SARS coronavirus (SARS-CoV), the viral load in COVID-19 (SARS-CoV-2) was higher and more often detectable by nasopharyngeal swabs and therefore active viral replication in the upper respiratory tract was suggested [1]. A prolonged viral shedding from the aerodigestive tract leads to a high and effective transmission of SARS-CoV-2, and impact on the control of infections. In several countries, the patients' care was unsustainable due to high amount of acute COVID19 infections, so that emergency departments and intensive care units (ICU) collapsed under the flood of patients. Therefore, several preparations are necessary, such as excellent communication, immediate triage of potentially infectious patients, definition of treatment and diagnostic algorithms to identify patients with risk factors as well as suitable protective devices for hospital personnel [3].

Since first reports of COVID-19 late in 2019 as an epidemic infection $[4,5]$, the spread of COVID-19 became a worldwide public health emergency, so that the World Health Organization (WHO) on 11 March 
2020declared COVID-19 as a pandemic [6]. As a consequence of this fact and increasing infection rates, the Swiss government as many other governments worldwide, decreed a public lock-down to reduce and restrict further COVID-19 infections on 13 March 2020 (effective as of 14 March 2020), by restraining the public life to avoid intense social contacts and to interrupt chains of infection.

Due to the severe spread of COVID-19 and to redistribute the manpower and hospital resources, elective surgeries were reduced or even cancelled except for specific urgent interventions [7, 8]. The main goal of this restriction was to maximize the hospital and ICU beds and to shift manpower towards diagnosis and acute treatment of COVID-19 patients. Furthermore, these acute changes in hospital utilization should reduce the unnecessary exposure of patients and healthcare workers with potentially positive but asymptomatic COVID-19 carriers.

There are reports that in some medical specialties, such as dermatology [9], orthopedics [7], cardiology [10], urology [11] and neurology [12] the treated emergency cases during the COVID-19 pandemic were reduced in comparison to prior time periods. A possible explanation for this fact might be that not the incidence of certain diseases decreased during that time, but patients were afraid of infection and avoided attending hospitals for further diagnosis and treatment [10]. Furthermore, reports from Spain and Hong Kong showed that in the case of a cerebral stroke not only the number of treated cases was reduced but also the arrival time and start of treatment was delayed [12, 13].

The aim of this retrospective investigation was to provide an overview on the impact of the COVID-19 lock-down on the presentations of patients in an interdisciplinary Swiss emergency department (ED). To the best of our knowledge, this is the first investigation, in a rapid evolving field of science, on the influence of COVID-19 public lock-down on the number of presentations in the emergency department of a large public hospital in Switzerland.

\section{Patients, material and methods}

A retrospective study was performed at an interdisciplinary Swiss ED with approximately 35,000 consultations per year. The spectrum of treated patients ranged from emergencies in general internal medicine including subspecialties (e.g. cardiology, nephrology, gastroenterology), surgery, orthopedics, traumatology, neurology, urology, gynecology, and otolaryngology.

During the governmentally instituted lock-down between 14 March 2020 and 26 April 2020, all patients who presented to the ED were enrolled in this investigation. As a control cohort all patients during the identical time period in 2019 were included in this analysis.
Data regarding patients' characteristics (age, gender), medical specialty (nonsurgical specialties including general internal medicine, cardiology, nephrology, neurology, ophthalmology; surgical specialties including general surgery, visceral surgery, orthopedics, thoracic surgery, vascular surgery, gynecology, otolaryngology, urology), time course of presentations during the observation period, outpatient or hospital admission were collected and compared between the two time periods. In the case of trauma there was a differentiation between surgery (head trauma, thoracic and abdominal trauma) and orthopedics (spinal trauma, trauma to the extremities including pelvis).

Our ED consists of a fast-track ED and an interdisciplinary ED. The differences between these units are the opening hours and the severity of diseases of the treated patients, which was assessed by the multilevel emergency severity index (ESI 1-5). The aim of this score is to assess the urgency of a treatment and the need of ED resources to triage patients to the suitable treatment level [14]. The fast-track ED mainly covers patients between 8.00 am to 11.00 pm with ESI 4 and 5 , whereas the interdisciplinary ED is open 24/7 covering patients with ESI triage level 1-3. The triage of patients was performed by an experienced and trained emergency department nurse. Furthermore, according to internal guidelines the triage to the medical speciality occurred during initial assessment but was clarified based on the final findings.

\section{Statistical analysis}

Continuous variables were reported as mean and standard deviation or median and interquartile range as appropriate. Comparison of the groups was performed using Student's t-test. Categorical variables were reported as proportions and compared with the $\chi^{2}$ and Fisher's exact test. Statistical analysis of the data and graphics were performed with GraphPad Prism 5.0 software package (GraphPad, San Diego, CA, USA), and a $p \leq 0.05$ was assumed as statistically significant.

\section{Results}

In total 7450 patients were included in this investigation. In addition, in a specific COVID-19 ambulatory setting off site of the ED, 994 patients were tested by nasopharyngeal swab for COVID-19 during the lockdown period. After exclusion of patients with missing data and patients under 18 years, 7072 patients were included in the final analysis. During the lock-down period the number of ED presentations significantly decreased by $29 \%$ in comparison to the control period in the previous year (2019 $n=4127$ vs. $2020 n=2945$; $p<0.0001)$. The decrease of consultations was even higher in the fast-track ED (2019 $n=1639$ vs. 2020 $n=875 ; 46 \%$ decrease; $p<0.0001$ ), than in the inter- 


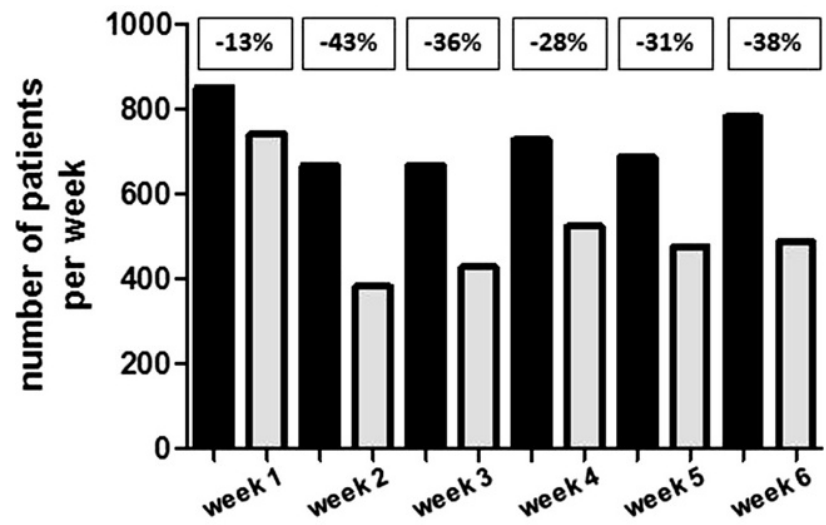

Fig. 1 Time course of ED presentations during the observation periods. Black columns 2019, grey columns 2020

disciplinary ED (2019 $n=2488$ vs. $2020 n=2070 ; 17 \%$ decrease, $p<0.0001)$.

The analysis of the time course during the lockdown of 6 weeks revealed that in each week the number of patients was lower during lock-down than in the corresponding week the year before (Fig. 1). In the second week the decrease was $43 \%$ and thus higher than in the other weeks and in the first week the smallest reduction was seen with $13 \%$.

Between the two time periods there were no statistically significant differences concerning age (2019 $52.1 \pm 21.4$ years vs. $202052.7 \pm 20.8$ years; $p=0.2$ ), or gender (2019 females $49.6 \%$ \% vs. 2020 females $49.9 \%$; $p=0.9$ ). There was no significant difference in the necessity of hospital admissions between the two time periods (2019 $n=1084,26.3 \%$ vs. $2020 n=829,28.1 \%$; $p=0.2)$.

Irrespective of the medical specialty, the number of presentations decreased during the COVID-19 situation. The decrease in presentations due to surgical diseases was $31 \%$ (2019 $n=1181$ vs. $2020 n=810$, $p<0.0001)$ and therefore much higher than in nonsurgical presentations with $3.6 \%$ (2019 $n=1307$ vs. 2020 $n=1260, p=0.4$ ).

\section{Discussion}

There was a significant decrease of presentations in the ED during the socioeconomic lock-down due to COVID-19 in Switzerland. This decrease was obvious in all investigated medical specialties, but mainly present in surgical diseases independent of COVID19.

The avoidance of many patients to refer to the hospital and potential infection with COVID-19 might be one possible explanation for this decrease; however, the decrease was seen in all medical conditions that require inpatient care and emergency treatment, as there were no differences in the necessity of hospital admissions. This fact leads to major concerns of the well-being of the general population during this lockdown situation.
Immediately after the governmental edict of social restriction, the numbers of ED presentations decreased, but the reduction was essentially obvious after week 2 and remained on the same level during the entire observation period. The reduction of presentations to our ED was even higher than in a previous report from England with 25\% [15]. Interestingly, the decrease of presentations to our fast-track ED was twice as high as in the interdisciplinary ED. Many patients treated in the fast-track ED are presenting medical issues, which can be considered trifles and often could be treated by a family physician. This reduction might be explained due to a general fear of getting infected with COVID-19 in a hospital and the recommendation to visit an ED only in severe alteration of the health condition to prevent an overload of hospital employees and health resources [15].

The impact of the reduction was more obvious with surgical presentations, which may be explained by a reduction of traffic accidents, work injuries, leisure accidents, and reduced violent crime incidence due to social distancing [16]. In addition, we observed a decrease of urgent general surgery procedures, such as acute appendicitis, perforated hollow viscus and abscess during the lock-down period in comparison to 2019 of about $29 \%$. Unfortunately, the numbers of each disease were too small to perform a valid statistical analysis. In contrast to the reduced numbers of patients with acute appendicitis, the rate of perforation was increased during the lock-down period, reflecting a possible delay in ED presentation. Unfortunately, we did not collect data of all patients regarding their clinical severity, so that a final conclusion, whether patients were more sick during the lock-down period in comparison to 2019 was not possible. Overall, we had no mortality in general or visceral emergency procedures in 2019 and during the lock-down period. The decrease of presentations was comparable in all surgical disciplines and was around 30\%. Nonsurgical presentations were not affected to the same extent, as during the lock-down period presentations due to COVID-19 associated symptoms and diseases, such as pneumonia, upper respiratory tract infections, examination for COVID-19 symptoms, directed patients to the ED.

The present study is limited by its focus on one isolated ED and the lack of inclusion of local family physicians' practices; however, anecdotal reports of some local family doctors revealed comparable effects of the COVID-19 lock-down.

\section{Conclusion}

The socioeconomic lock-down impacted the number of presentations in a Swiss ED. Mainly, surgical diseases and injuries were affected in terms of a reduction of presentations during the COVID-19 period. Whether this effect will remain after COVID-19 and 
impact the general morbidity and mortality rate in Switzerland needs to be analyzed in the future.

Author Contribution All authors contributed to the study conception and design. Material preparation, data collection and analysis were performed by René Fahrner, Stefan Bähler, and Gregor Lindner. The first draft of the manuscript was written by René Fahrner and all authors commented on previous versions of the manuscript. All authors read and approved the final manuscript.

\section{Compliance with ethical guidelines}

Conflict of interest G. Lindner discloses potential conflicts of interest (consultant fees and fees from Bayer, Fresenius Kabi, Otsuka, Daiichi Sankyo; travel grants from GlaxoSmithKline, Otsuka, and Pierre Fabre). R. Fahrner and S. Bähler declare that they have no competing interests. None of the authors obtained funding for this specific report.

Ethical standards Ethics committee approval was obtained for this retrospective study from the Ethics Committee of North-West and Central Switzerland (No. 2020-01359) and the need for individual patient consent was waived.

\section{References}

1. Wolfel R, Corman VM, Guggemos W, et al. Virological assessment of hospitalized patients with COVID-2019. Nature. 2020; 581(7809):465-9. https://doi.org/10.1038/ s41586-020-2196-x. Epub 2020 Apr 1.Nature. 2020. PMID: 32235945.

2. Hoffmann M, Kleine-Weber H, Schroeder S, et al. SARSCoV-2 cell entry depends on ACE2 and TMPRSS2 and is blocked by a clinically proven protease inhibitor. Cell. 2020;181(e8):271-280e8.

3. Mockel M, Bachmann U, Behringer W, Pfafflin F, Stegemann MS. How emergency departments prepare for virus disease outbreaks like COVID-19. Eur J Emerg Med. 2020;27:161-2.

4. Zhu N, Zhang D, Wang W, Li X, Yang B, Song J, et al. A Novel Coronavirus from Patients with Pneumonia in China, 2019. The New England journal of medicine. 2020;382(8):727-33. PubMed PMID: 31978945. Pubmed Central PMCID: 7092803.

5. CoronaviridaeStudy Group of the International Committee on Taxonomy of V.. The species Severe acute respiratory syndrome-related coronavirus: classifying 2019-nCoV and namingitSARS-CoV-2. Nat Microbiol. 2020;5:536-44.

6. Cucinotta D, Vanelli M. WHO Declares COVID-19 a Pandemic. Acta Biomed. 2020;91:157-60.
7. Nunez JH, Sallent A, Lakhani K, et al. Impact of the COVID19 pandemic on an emergency traumatology service: experience at a tertiary trauma centre in Spain. Injury. 2020;51(7):1414-8. https://doi.org/10.1016/j.injury.2020. 05.016. Epub2020 May 13.Injury. 2020. PMID: 32405089.

8. Hemingway JF, Singh N, Starnes BW. Emerging practice patterns in vascular surgery during the COVID-19 pandemic. JVasc Surg. 2020;72(2):396-402. https://doi.org/10.1016/j. jvs.2020.04.492.

9. Tartari F, Guglielmo A, Fuligni F, Pileri A. Changes in emergency service access after spread of COVID19 across Italy. J Eur Acad Dermatol Venereol. 2020;34(8):e350-1. https:// doi.org/10.1111/jdv.16553. Epub 2020 Jun 4.J Eur Acad Dermatol Venereol. 2020. PMID: 32339340.

10. Toniolo M, Negri F, Antonutti M, Mase M, Facchin D. Unpredictable fall of severe emergent cardiovascular diseases hospital admissions during the COVID-19 pandemic: experience of a single large center in northern Italy. J Am Heart Assoc. 2020;9(13):e017122. https://doi.org/10.1161/JAHA. 120.017122. Epub 2020 May 22.J Am Heart Assoc. 2020. PMID: 32441548

11. Porreca A, Colicchia M, D'Agostino D, et al. Urology in the time of Coronavirus: reduced access to urgent and emergent urological care during the Coronavirus disease 2019 outbreak in Italy. Urol Int. 2020;104(7-8):631-6. https:// doi.org/10.1159/000508512. Epub 2020 May 20.Urol Int. 2020. PMID: 32434207.

12. Rudilosso S, Laredo C, Vera V, et al. Acute stroke care is at risk in the era of COVID-19: experience at a comprehensive stroke center in Barcelona. Stroke. 2020;51(7):1991-5. https://doi.org/10.1161/STROKEAHA.120.030329. Epub 2020 May22.Stroke. 2020. PMID: 32438895.

13. Teo KC, Leung WCY, Wong YK, et al. Delays in stroke onset to hospital arrival time during COVID-19. Stroke. 2020;51(7):2228-31. https://doi.org/10.1161/ STROKEAHA.120.030105. Epub 2020 May 20.Stroke. 2020. PMID: 32432998.

14. Grossmann FF, Delport K, Keller DI. Emergency severity index. Translation of a valid instrument into german. Notfall Rettungsmed. 2009;12:290-2.

15. Thornton J. Covid-19: A\&E visits in England fall by $25 \%$ in week after lockdown. BMJ. 2020;369:m1401.

16. Kocher KE, Arora R, Bassin BS, et al. Baseline performance of real-world clinical practice within a statewide emergency medicine quality network: the Michigan emergency departmentimprovement collaborative (MEDIC). AnnEmerg Med. 2020;75:192-205.

Publisher's Note Springer Nature remains neutral with regard to jurisdictional claims in published maps and institutional affiliations. 